\title{
Active Knowledge for the Situation-driven Control of Product Definition
}

\section{László Horváth and Imre J. Rudas}

Óbuda University, John von Neumann Faculty of Informatics, Institute of Applied Mathematics; Bécsi út 96/b, H-1034 Budapest, Hungary

e-mail: horvath.laszlo@nik.uni-obuda.hu,rudas@uni-obuda.hu

\begin{abstract}
The current product model consists of features and unstructured contextual connections in order to relate features. The feature modifies the previous state of the product model producing contextual connections with previously defined features. Active knowledge is applied for the adaptive modification of product model features in the case of a changed situation or event. Starting from this state-of-the-art, the authors of this paper introduced a new method to achieve higher-level and more advanced active feature driven product model definition. As part of the related research program, new situation driven model definition processes and model entities are explained in this paper. Higher-level knowledge representation in the product model is motivated by a recent trend in industrial product modeling systems towards more advanced and efficient situation-based selfadaptive model generation. The proposed model represents one of the possible future ways of product model development for product lifecycle management (PLM) systems on the global or product level of decisions. Its implementation will be new application-oriented model entity generation and representation utilizing existing modeling resources in industrial PLM systems by use of application programming interfaces (API).
\end{abstract}

Keywords: product model; situation-based product definition; active knowledge in product model; product behavior driven feature definition

\section{Introduction}

The work of engineers has been supported by computer applications for a long time. Digital drawing and documentation representations in the early years of computer-supported engineering were changed to geometric and then to product modeling during the eighties and nineties. Nowadays, the product model has the capability to represent arbitrary engineering objects together with their relationships. The trend towards virtual engineering space [1] can be recognized easily in current developments in order to represent all characteristics of a planned product and its connections. This paper introduces some results about a new concept, approach and method as a contribution towards achieving a complete and 
consistent model for virtual engineering space. According to this concept, the communication between engineer and model entity generation must be raised from the present product feature definition level to the function and knowledge request level. The approach covers communication of this request as human intent, its harmonization with other intents, and its repeated application for a changed situation and event in order to assist decisions on product features. For this purpose, the method is being developed for request definition and processing in the product model in the form of the appropriate structure of new features.

The proposed modeling is intended to realize the organic extension of current product modeling in product lifecycle management (PLM) systems towards better human communication and decision making. The results of the research are planned to be implemented experimentally into an open PLM system where application programming interface (API) is available for the programming of modeling in the application environment and to provide access to existing features, modeling procedures, and user surfaces in that PLM system. This also facilitates the strong application orientation of industrial product definition.

This paper is an extended and upgraded version of a contribution to the proceedings of the 6th International Workshop on Knowledge Management [2]. It starts with an introduction to the achievements in product modeling that are in close connection with the proposed function level human request based feature definition. The proposed product modeling extends the currently applied product modeling. A detailed introduction of the currently prevailing and well proven engineering technology is impossible within the scope of this paper. The reader is supposed to know recent advanced feature-based and knowledge-ware-driven contextual modeling in the PLM environment.

After an introduction to the currently prevailing advanced product modeling and its model representation capabilities, the knowledge processes in the current and proposed product definition are compared. Then the replacement of the current formal knowledge and object parameter value communication with the proposed influence request communication between engineer and product feature generation process is explained. Following this, current product model entities, including influence requests, actual objective structure, product feature context structure, and actual behavior structure, are detailed. Finally, affect analysis along contextual chains propagating a change of product feature is explained.

\section{Preliminaries and Purpose}

Engineering practice is moving into product lifecycle management (PLM) systems. A PLM system represents a high level of product information integration. The trend in this integration have led to complex and large product models with a very high number of entities and a crowd of unorganized relationships. Work in 
this paper was motivated by the recognition that the definition and processing of this current product model need more organized relationships with more efficient knowledge representation. In an earlier work [3], it was concluded that background content controlled engineering object definition would be required for product development in model space. The proposed new method of function level human request based feature definition is devoted to realizing this concept. It is grounded on former works in the modeling of engineering intent [4], the definition of product behavior in the product model [5], knowledge representation in the product model [6], and information content based product modeling [7]. Change propagation to engineering objects and adaptive action was described in [8]. These publications cover different aspects of knowledge based engineering modeling and lay the groundwork for the development of the method introduced in this paper.

The term 'product model' is applied for the definition of consistent engineering purposed computer representation of the product. It was grounded theoretically during development of the STEP (Standard for the Exchange of Product Model Data, ISO 10303) product model standard by the International Standards Organization (ISO). This is the only standard for a product model and is under implementation in different industries by using application protocols. Knowledge related engineering research works generally consider STEP. As a representative example, a framework was proposed for integrated data and knowledge model, using reference protocols in [9].

A step-by step organic development of engineering modeling and simulation has led to the present product lifecycle management (PLM) technology. In order to identify the place of the proposed modeling in product modeling, the development of engineering modeling and simulation is tracked in Fig. 1. Geometry was the first area of intensive research because mathematic description of shape was urged by the early control of machine tools and robots. Nowadays, the only representation of curves and surfaces in PLM systems is the sophisticated nonuniform rational B-spline (NURBS). The connection of knowledge and geometry for higher quality shapes is also a concern in the proposed modeling. On this topic, paper [10] introduces an application of knowledge-guided NURBS at repair imported incompatible geometric models. Boundary representation (B-rep) was involved in geometric modeling in order to complete geometry by topology for the representation of geometric structure. Euler operators are applied to establish the topological structure of the solid body using vertex, edge, and face entities, while geometry is defined during construction of the solid shape in the model space. In the seventies, a breakthrough in simulation technology was the application of finite element modeling and analysis (FEM/FEA). This facilitated the approximation of location dependent parameters at nodes in a mesh generated on a geometric model. By now, mesh is associative with geometry, parametric, and adaptive. FEM/FEA fits well into the knowledge based product model. By active application of FEM/FEA, design variables acting on product performance can be controlled. B-rep and FEM/FEA are essential elements of the current product definition in PLM systems. 
The application and variant orientation of a product model is allowed by feature and parametric modeling principle, respectively. These principles and the related modeling achievements are in close connection with development of the proposed modeling concept, approach, and method. Form feature is an application oriented shape aspect and uses B-rep representation. The definition of relative placing and the moving of solids in model space represent placing constraints and degrees of freedom, respectively. Feature based product modeling is still a frequent area of research. In related research [11], parametric and semantics based feature is defined in order to support function-oriented product modeling together with better communication in an engineering modeling environment. Currently, the feature principle has been extended to all entities in the product model. During the nineties, parametric modeling allowed for the definition of dimensions as variables. The parameter model was introduced to represent the relationships among dimensions. In current product models, the arbitrary parameter can be defined and related.

This century started with two new essential techniques in product modeling. They are contextual modeling and knowledge ware. In contextual modeling, feature parameters are to be defined in the context of other feature or product level parameters. Knowledge ware is applied for the collection of local or corporate knowledge in order to facilitate situation and event based control of feature generation. Among others, knowledge ware includes representation of relationships and functional connections of parts and their features as well as related algorithms.

The development of knowledge ware driven product entity generation placed emphasis on knowledge management in PLM systems. The personalization and codification in knowledge management in engineering design are introduced for the development of a multidisciplinary framework in [12]. Paper [14] introduces an approach to the definition and mapping of expert knowledge. In [15], functional behavior and structure model is introduced as design conceptual space. Function behavior and structure model, and design process schema are suggested as way to understand the typical life cycle of a decision-making process in design. All knowledge sources are involved in this conceptual model. In [16], knowledge acquisition processes are defined to capture structured knowledge systematically, and knowledge representation technology is characterized to store knowledge, preserving important relationships. The objective is to establish a better solution than conventional engineering data bases. Paper [17] shows multiple expert inputs in knowledge learning. Input is processed by the Bayesian Network into a structure. Empirical data is applied to refine and parameterize this structure. The product model is in strong contextual connection with business processes. In [13], a method is shown for P-graph based workflow modeling of business processes. The above knowledge methodology developments are efforts to solve problems produced by the emerging knowledge based PLM. Modeling in this paper is devoted to a global solution on product level that can utilize the above methods at local level problem solving. 


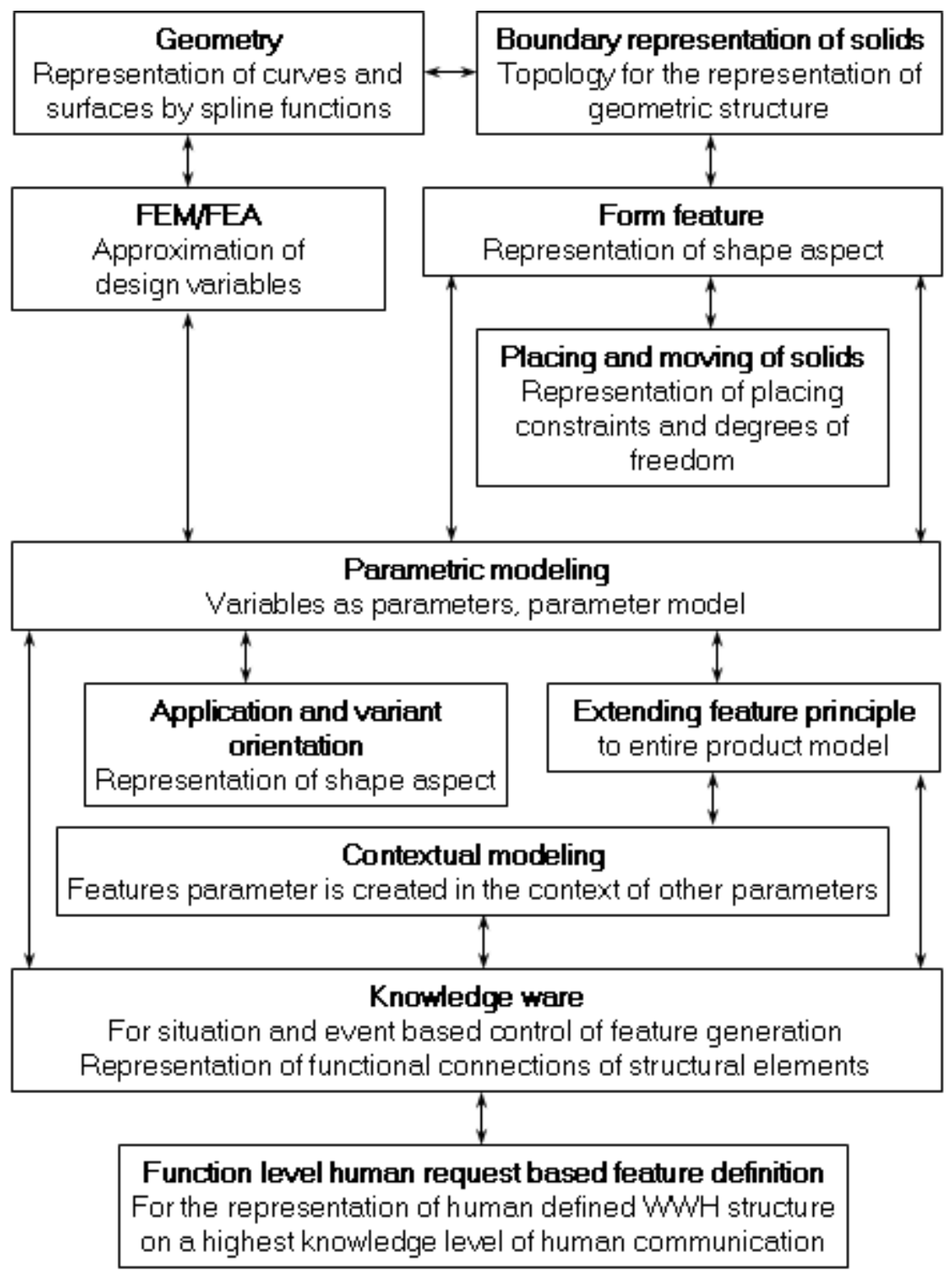

Figure 1

Step-by-step development of engineering modeling

The above characterization and summary of currently prevailing product modeling introduce the modeling proposed in this paper. The proposed modeling, called Function level human request based feature definition, is the result of efforts to bring product definition dialogue closer to human thinking. On a higher 
knowledge level of the human-modeling process communication, the human defines advanced model features in order to contribute via model representation of why, what, and how (WWH). The human requires product functions together with demanded quality and specified or proposed methods in a contextual structure. A decision on product feature definition is based on coordinated requests of authorized humans. It results in affect analyzed feature definition and applies adaptive actions for the control of product feature generation.

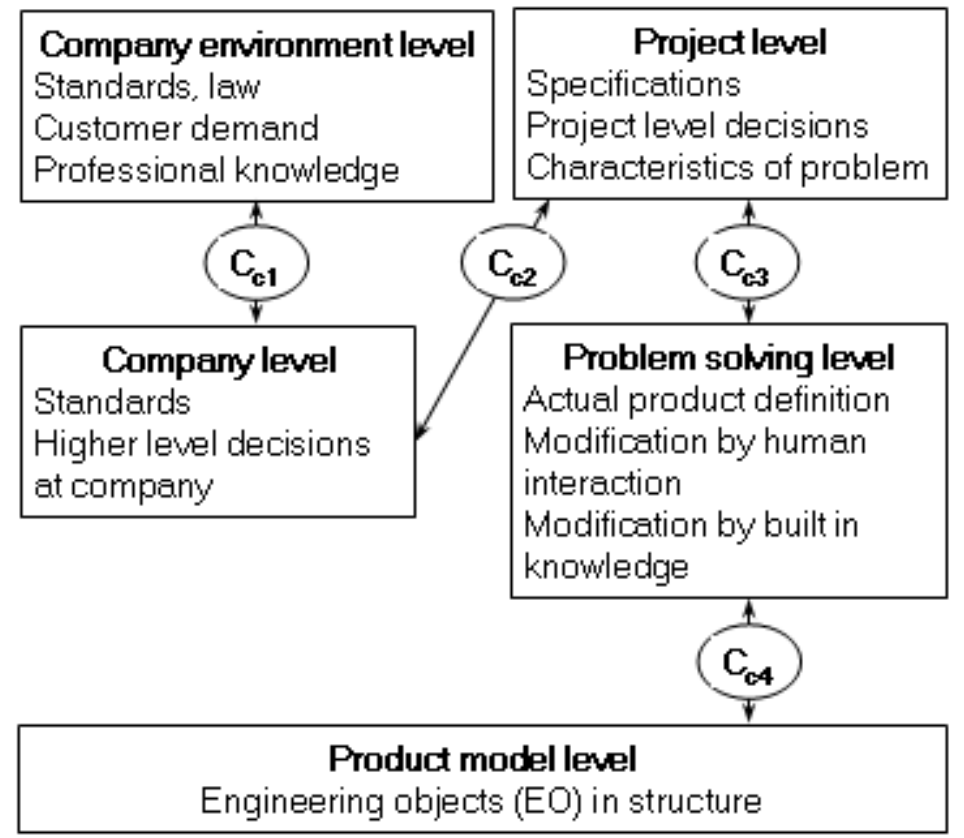

Figure 2

Levels in an engineering system

Decisions on engineering objects for a product are made in the context of relevant knowledge. Knowledge is defined on company environment, company, project, problem solving, and product model levels (Fig. 2). These levels constitute a contextual chain. On the company environment level, standards, laws and customer demand, as well as professional knowledge, are defined. Company level knowledge is defined in the context of company environment level knowledge $\left(\mathrm{C}_{\mathrm{c} 1}\right)$ and represents company standards and higher level decisions. Project level knowledge is defined in the context of company level knowledge $\left(\mathrm{C}_{\mathrm{c} 2}\right)$ and represents specifications, project level decisions, and the characteristics of problems. Problem solving level knowledge is defined in the context of project level knowledge $\left(\mathrm{C}_{\mathrm{c} 3}\right)$ and represents actual product definition, modification by human interaction, and modification by built in knowledge. Finally, the product model level includes engineering objects (EO) in an appropriate structure and is 
defined in the context of the problem solving level $\left(\mathrm{C}_{\mathrm{c} 4}\right)$. The modeling in this paper is proposed for the problem solving level. The trend to include higher level knowledge and specification in contextual chains within product model was considered.

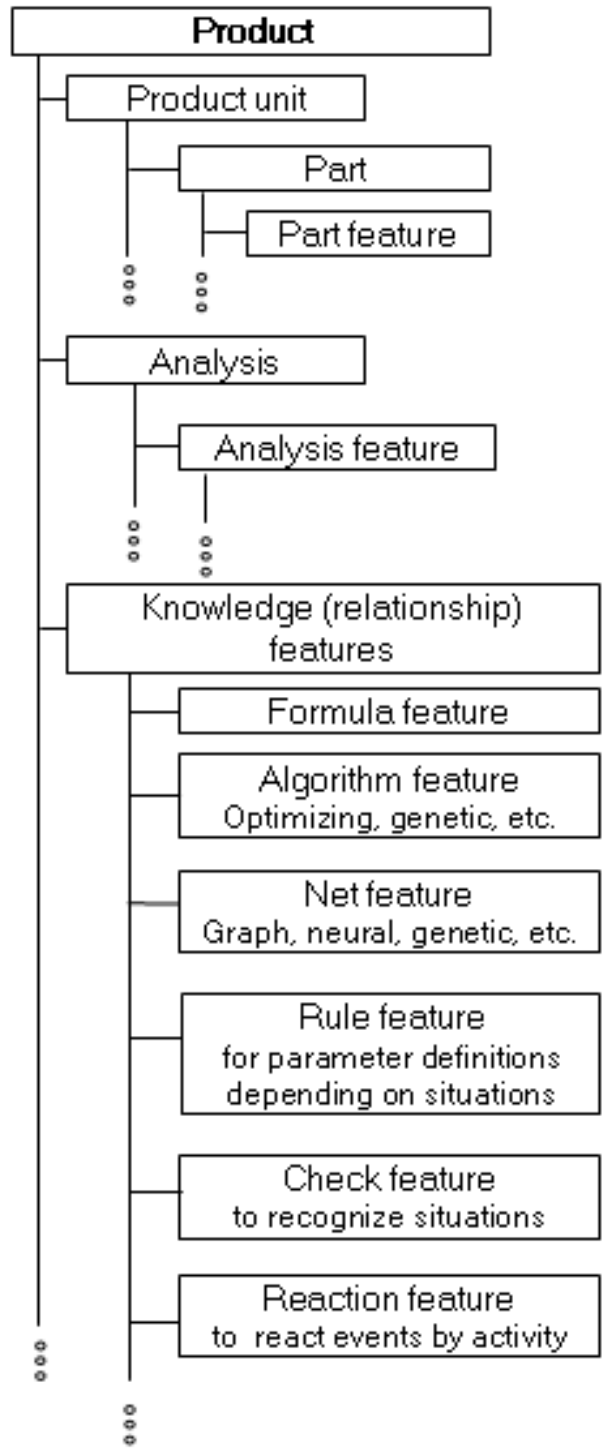

Figure 3

Product model level with engineering objects in structure 


\section{Current Practice}

Including active knowledge representations in the product model is one of the most important trends in the current developments of product modeling. Purposeful knowledge features are available in PLM systems for the current product model.

Product objects as features are organized into units in the structure of the product model according to the model representation demanded by the actual product (Figure 3). Analysis and knowledge features are separated from these units in the structure for organizational and acceptance causes.

Simple, engineer-understandable but efficient knowledge representations are preferred by engineers. The formula is free to define as a means of context definition for product and other features. Optimizing, genetic, and other algorithms make user development of product modeling possible. Connectivity knowledge is included in the product model by various graphs, neural networks, genetic support, and other connective entities. The active definition and the checking of engineering objects are driven by rules, checks, and reactions (Fig. 3). When a rule, a reaction, or value of its parameter is defined or changed, the related engineering objects are changed automatically by the execution of the rule or reaction. Similarly, check notices change request and offers change for the relevant engineering objects. Check and rule are involved for handling situations, while reaction for handling events in product model. Check is applied to recognize the situation, whereas rule is applied for the parameter definition depending on the situation. Reaction activates predefined activity for new or changed event.

The current product model (Fig. 3) is considered in this paper as a classical way of product definition. It represents a well-proven principle, method and system background. Modeling in this paper is built as an extension to this strong basis. The knowledge process in the current or classical product definition was analyzed in order to identify knowledge-related problems during model construction and communication amongst engineers. In Fig. 4, two engineers communicate through a product model. One of the engineers is in interaction with the product feature definition processes. The knowledge background is available for human activities. This background is available as a representation or should be represented in the product model. However, a high proportion of knowledge is applied by the human but not communicated with the modeling system, because no capability is available for representation. The engineer communicates knowledge and object parameter values. Product feature generation processes generate features for the product model and place them in a structure. Similarly to human communication, a high proportion of knowledge is applied by processes, but no capability is available for representation in the product model. The other engineer is in interaction with product feature application processes. Due to the above shortage in knowledge representation capabilities, this engineer is not properly informed. 
Information is not included in the product model about the process to a decision, allowed modifications, the strength of a decision, and some of the relationship definitions. The main purpose of the modeling proposed in this paper is to fill this gap caused by a lack of knowledge in the dotted boxes in Fig. 4.

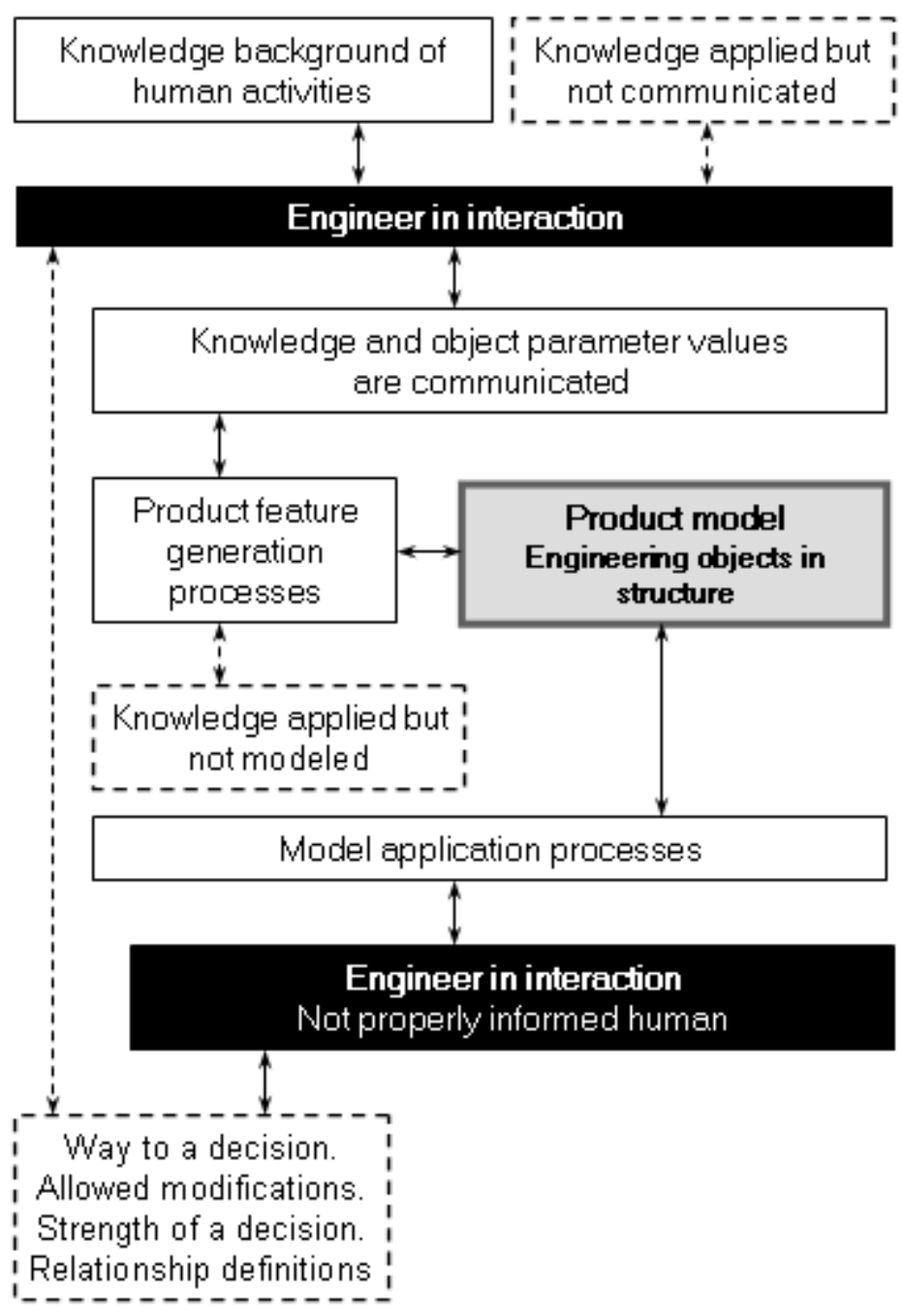

Figure 4

Knowledge process in current product definition 


\section{The Proposed Product Definition}

Starting from the knowledge process in the current product definition, the extension to knowledge representation and its application for product model feature generation are introduced in Fig. 5. Beyond the knowledge background of human activities, outside expert sources and activities are to be connected to the modeling in order to allow for any knowledge to be included in the product model. Depending on the feature choice available in a PLM system, extensive knowledge representation in the product model is a real possibility in future engineering. Theoretically, lost knowledge is not allowed. Otherwise, the self-adaptive product model cannot work properly. However, knowledge that intentionally is not communicated or cannot be represented in the product model may be allowed to be applied by an engineer only in interaction. The resulted feature parameter values are applied as constraints on those can be modified according to coordination requests from different engineers. Constraining and contextual connection handling functionality in the current product definition is utilized at the proposed knowledge feature definition.

In the proposed method, knowledge and object parameter value communication between engineer and product feature generation processes are replaced by influence, or in other words, by product definition request communication (Fig. 5). Influence requests are represented for product model. Different requests for the same features are coordinated, and an actual influence definition is generated. Model feature generation always works by using the actual influence definition and modifies the affected features when this definition is modified. Compliance of the actual influence definition with the existing product model is evaluated by affect analysis. Product feature generation processes are controlled by using product behavior definitions. Consequently, any request against product feature generation must be defined as product behavior. For this reason, an extended definition of behavior was proposed in [5].

Extended model application processes communicate information carried by extension entities in the product model with an engineer who applies product model information. This engineer is a properly informed human depending on the model representation provided by the implementation of the extension. The product model to be extended includes engineering objects in product structure and product function structure as the main relevant elements of the current product model (Fig. 5). The extension involves new entities for the new modeling tasks including influence requests, actual objective structure, product feature context structure, and actual behavior structure. These entities will be detailed and explained in the rest of this paper (Figs. 6 and 7). 


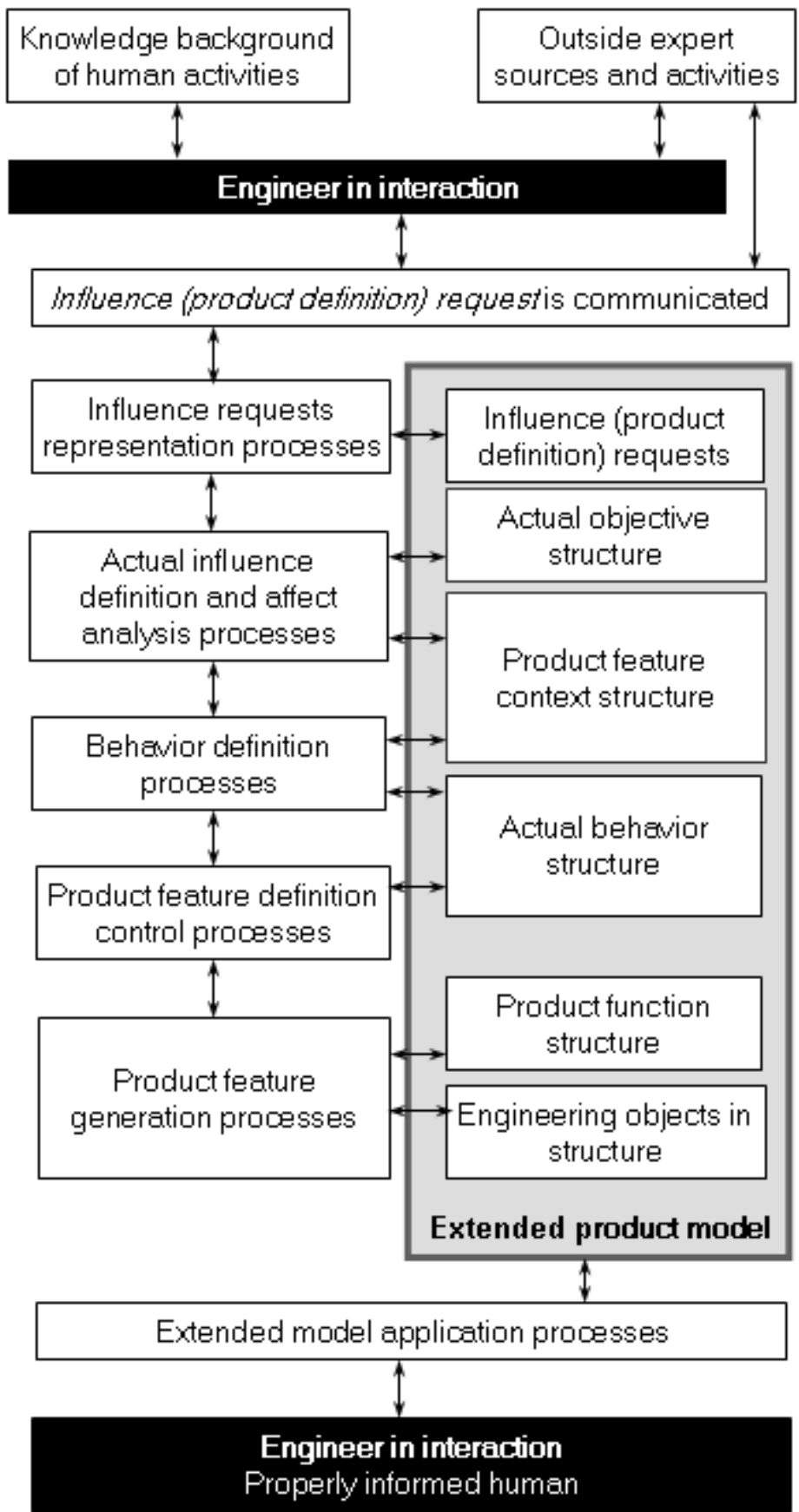

Figure 5

Knowledge process in the proposed product definition 


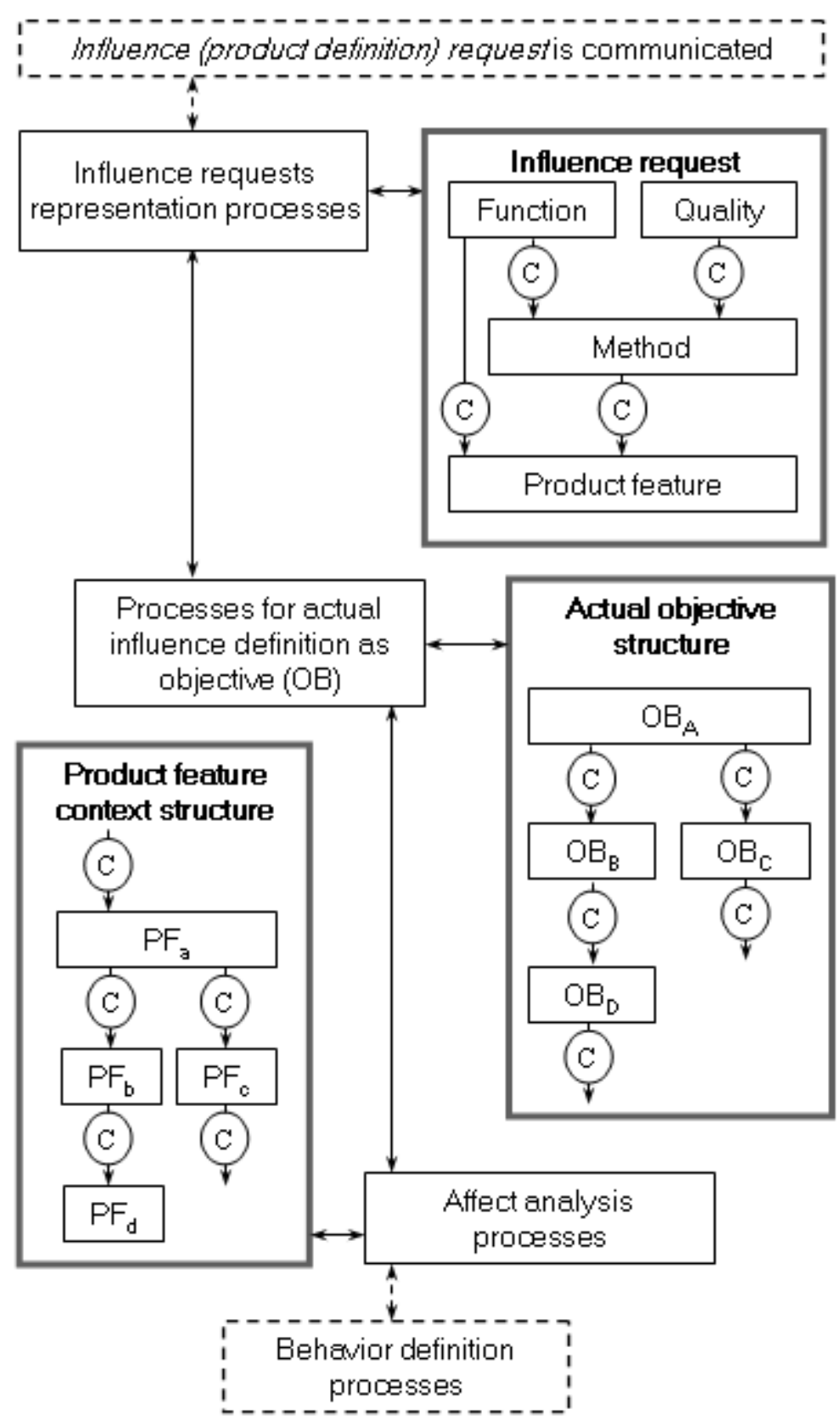

Figure 6

Influence request and objective 


\section{New Product Model Entities}

The new entities are represented as features for the proposed modeling and are in appropriate contextual connection with current product model features. The influence request is communicated with the appropriate processes in the extension and then processed into an influence request feature.

The influence request feature is contextual with the function to be realized, the quality to be kept, the method to be applied, and the product features to be generated for the product under development (Fig. 6). Letter ' $\mathrm{C}$ ' in a circle symbolizes a contextual connection entity, while the arrow shows the direction of context.

The method for the definition of the product features is defined in the context of function. Quality is a specification for the awaited function related characteristics of the product. A product feature is defined in the context of function and quality. Entity contextual connection is free to be defined using a wide range of tools available in PLM systems, such as formula, function, algorithm, rule, etc. The well proven construction tools are available in the PLM system.

Product feature generation is controlled by the actual behavior structure (Fig. 7). Behavior provides objective driven product feature generation instead of the current conventional direct product feature generation. The behavior feature will be detailed and explained in the rest of this paper (Fig. 8). The behavior definition is assisted by the situation and circumstance set definition.

In the background of a behavior, the situation collects circumstances as product functions, objects, parameter definitions, etc. and provides all information to be considered at the product object definition. The control of object generation is governed by the use of information in adaptive action. Adaptive action is also handled as a feature and it is made ready to execute by the appropriate decision on product objects.

The main contextual connections in the product model extension constitute contextual main chains, as shown in Fig. 8. The function in the product function structure and the objective in the actual objective structure are defined in the context of the influence request. The product function structure connects product features by functions and it is available in current advanced product models. Behaviors are organized for the product in the actual behavior structure. This structure includes behaviors $(\mathrm{BH})$ connected by contextual definitions. Behavior in the actual behavior structure is defined in the context of the relevant objective in the actual objective structure, and it also depends on the contextual connections inside of this structure. Basically, behavior is defined as a contextual chain of behavior (as defined), situation, circumstances, and adaptive action. Product features and their contextual connections are defined in the context of adaptive action. At the same time, engineering objects in their structure are generated in the 
context of product features and their contextual connections in product feature context structure.

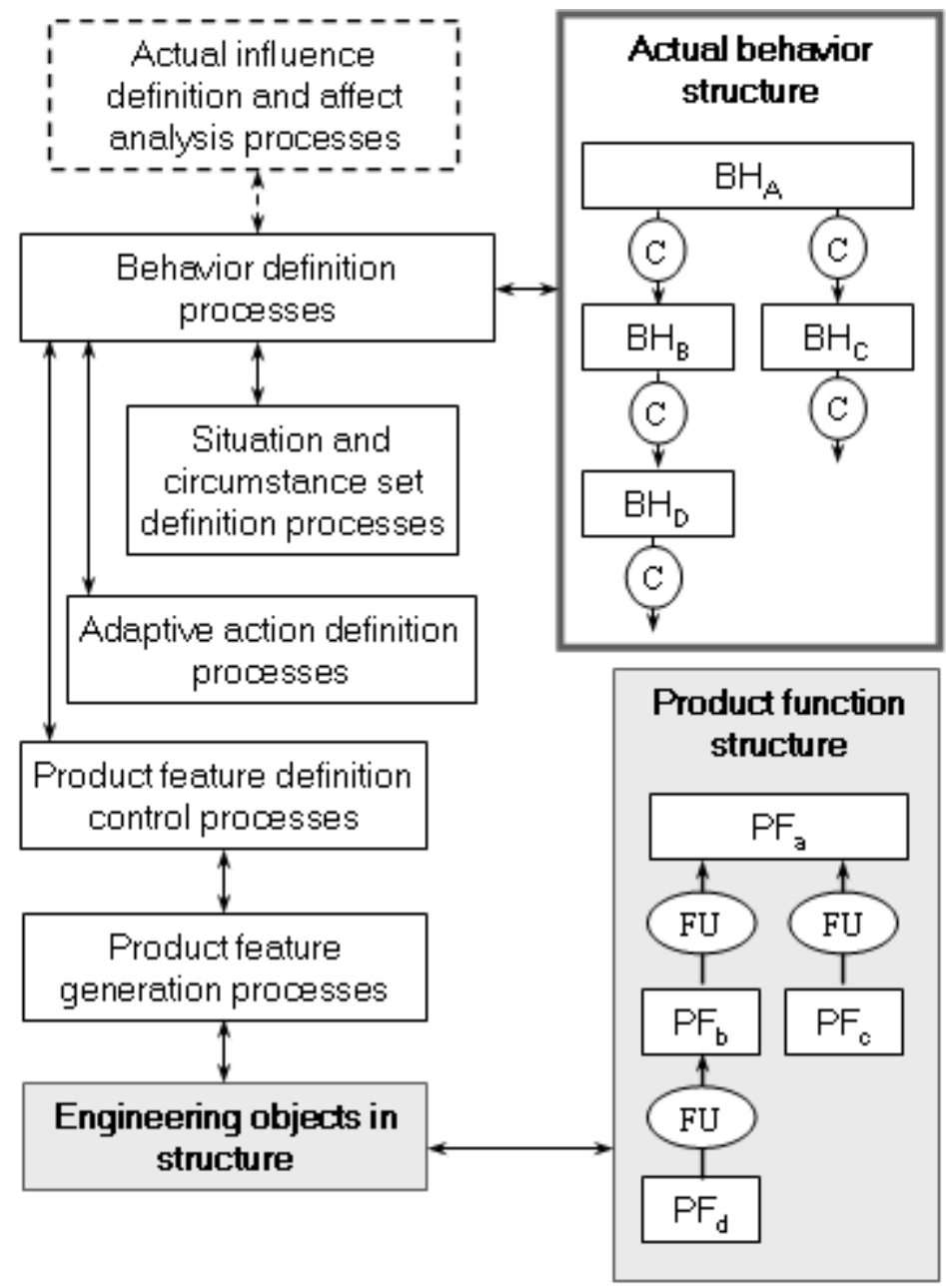

Figure 7

Behavior and control of product feature generation

Influence requests from different engineers in the PLM system are synthesized into an actual objective structure. Parallel and overlapping influence definitions are decided and harmonized into actual influence definitions, as was conceptualized in [7]. These definitions are handled as objectives. The elaboration of the methods for these modeling functions is a future plan. At this time, the application of dialogues provided by current PLM systems is considered. The actual objective structure consists of objectives and their contextual connections. As was stated above, a lack of organized contexts in the current product model 
requires a special feature for the product feature context structure in the product model extension. This structure consists of product features (PF) and their contextual connections and is used via affect analysis processes. Affect analyzed and corrected actual objective structure is applied by behavior definition processes.

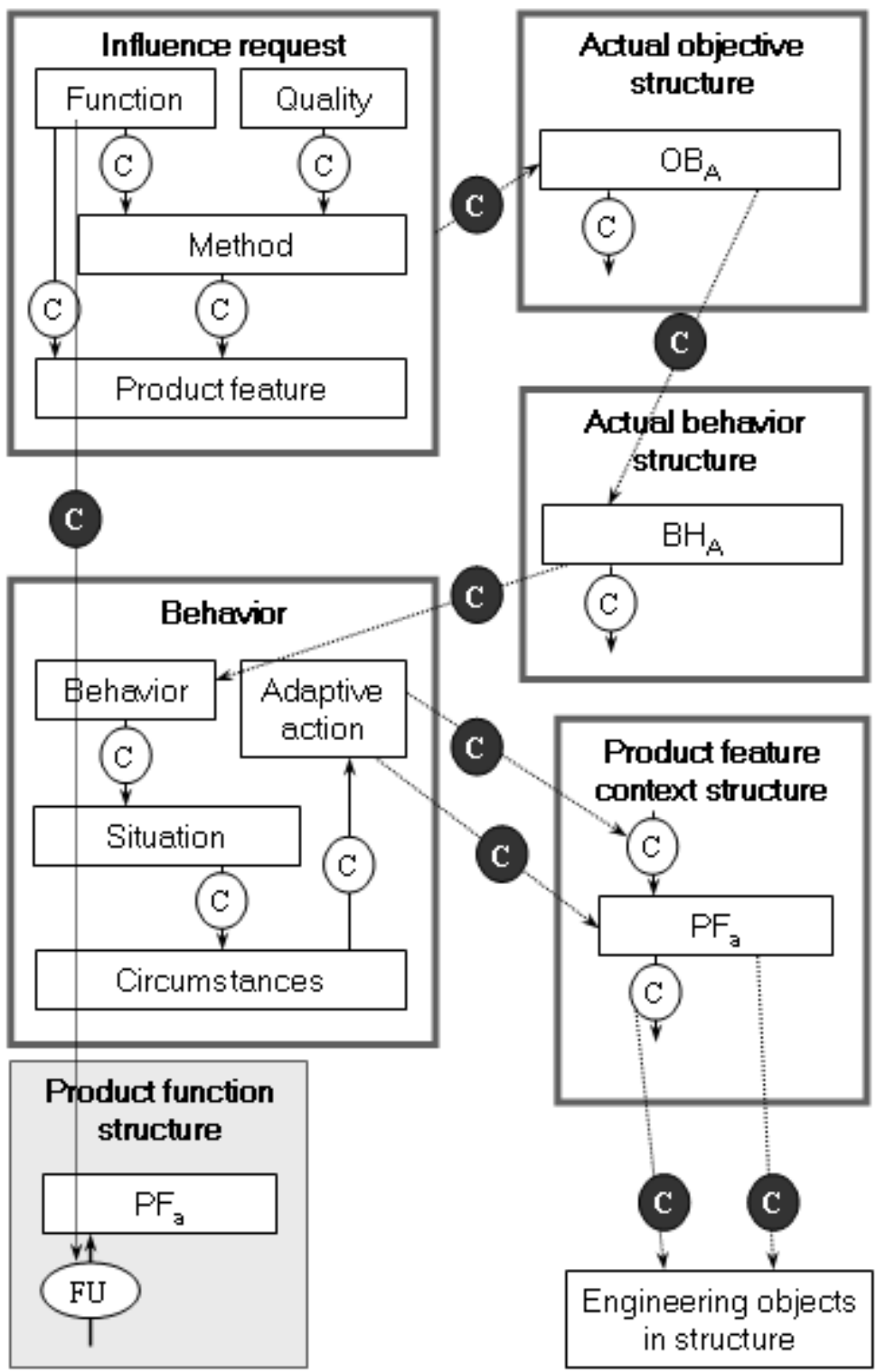

Figure 8

Main contextual connections in the extension of product model 


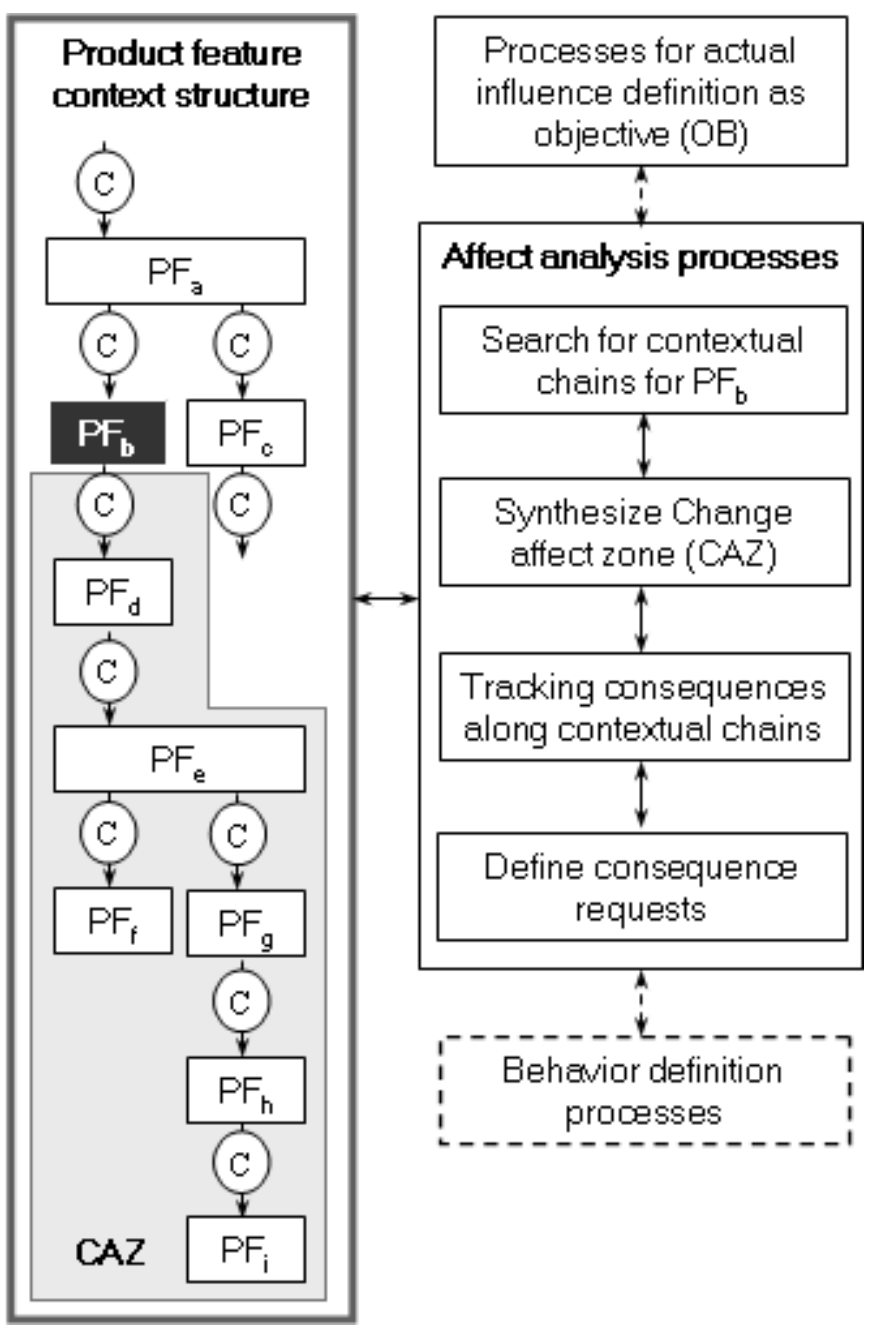

Figure 9

Affect analyses in CAZ

Analysis for the affects of actual objectives on existing or already decided product objects is done, as explained in Fig. 9. Affect analysis is done along the contextual chains propagating change of a product feature through other product features. In Fig. 9, this product feature is $\mathrm{PF}_{\mathrm{b}}$. These chains are searched in the change affect zone (CAZ). The CAZ includes all potentially affected product features and synthesizes contextual product feature chains. Tracking consequences along the contextual chains results in consequence requests to be included in the product model extension. Objectives and behaviors must be updated accordingly. 


\section{Conclusions}

This paper introduces new results in modeling methodology and related model entities in order to enhance knowledge communication between human and product feature generation processes and to establish an organized knowledge background of the product feature definition in the product model. The currently applied and well-proven product modeling technology is extended. The wellestablished direction of development of this modeling technology makes continuous development of the proposed extension to the currently applied modeling possible

Due to a shortage in the knowledge representation capabilities of the current product modeling, information about the process to a decision, allowed modifications, the strength of a decision, and some relationship definitions cannot be included in the product model. The proposed extension applies influence request for communication between human and model generation, active objective structure for representation of coordinated and organized objectives of product development, actual behavior structure to represent new features for the control of product feature generation. The work has relied upon earlier results by the authors in this area of engineering modeling.

\section{Acknowledgement}

The authors gratefully acknowledge the financial support by the Óbuda University research fund.

\section{References}

[1] Horváth, L., Rudas, I. J.: Product Definition in Virtual Space Using Background information at Description of Engineering Objects, in WSEAS Transactions on Systems and Control, ISSN 1991-8763, Volume 3, Issue 9, 2008, pp. 748-758

[2] Horváth, L., Rudas, I. J.: Knowledge Representations for the Practice of PLM Systems, in proc. of the $6^{\text {th }}$ International Workshop on Knowledge Management, Trencin, Slovakia, 2011, pp. 65-76

[3] Horváth, L., Rudas, I. J.: Modeling of the Background of Human Activities in Engineering Modeling, Proc. of the $27^{\text {th }}$ Annual Conference of the IEEE Industrial Electronics Society, Denver, Colorado, USA, 2001, pp. 273-278

[4] Horváth, L., Rudas, I. J.: Intelligent Human-Computer Communication of Engineers at Extended Companies, in Journal of Advanced Computational Intelligence and Intelligent Informatics, Volume 10, Number 4, 2006, pp. 510-516

[5] Horváth, L., Rudas, I. J.: Adaptive Modeling for Robot Systems Based on Behaviors and Feature Driven Shape Descriptions, in WSEAS Transactions on Information Science and Applications, Volume 11, Number 2, 2005, pp. 1761-1771 
[6] Horváth, L., Rudas, I. J.: Knowledge Technology for Product Modeling, Chapter 5 of the book Knowledge in Context - Few Faces of the Knowledge Society, Walters Kluwer, 2010

[7] Horváth, L., Rudas, I. J.: Engineering Objective Driven Product Lifecycle Management with Enhanced Human Control, In book Towards Intelligent Engineering and Information Technology, in Series: Studies in Computational Intelligence, Vol. 243, Springer-Verlag, Berlin, Heidelberg, 2009, pp. 543-556

[8] Horváth, L.: New Design Objective and Human Intent Based Management of Changes for Product Modeling, Acta Polytechnica Hungarica, Volume 4, No. 1, 2007, pp. 17-30

[9] Jardim-Goncalves, R. Figay, N. Steiger-Garcao, A.: Enabling Interoperability of STEP Application Protocols at Meta-Data and Knowledge Level. International Journal of Technology Management, Volume 36, Number 4, 2006, pp. 402-421

[10] Rajab, K., Piegl, L.A., Smarodzinava, V.: CAD Model Repair Using Knowledge-guided NURBS, Engineering with Computers, Volume 28, Number 2, 2012, pp. 1-10

[11] Bronsvoort, W. F., Bidarra, R., Nyirenda, P. J.: Developments in Feature Modeling, Computer-Aided Design and Applications, Volume 5, No. 3, 2006, pp. 655-664

[12] McMahon, C., Lowe, A. Culley, S.: Knowledge Management in Engineering Design: Personalization and Codification, Journal of Engineering Design, Volume 15, Number 4, 2004, pp. 307-325

[13] J. Tick: Visualization and Simulation Tool for Analyzing P-Graph-based Workflow Systems, in Applied Computational Intelligence in Engineering and Information Technology, Springer-Verlag, Berlin Heidelberg 2012, pp. $71-82$

[14] Renaud, J.: Improvement of the Design Process through Knowledge Capitalization: an Approach by Know-how Mapping, Concurrent Engineering, Volume 12, Number 1, 2004, pp. 25-37

[15] Colomboa, G., Moscaa, A., Sartori, F.: Towards the Design of Intelligent CAD Systems: An Ontological Approach, Advanced Engineering Informatics, Volume 21, Number 2, 2007, pp. 153-168

[16] Preece, A., Flett, A., Sleeman, D., Curry, D, Meany, N., Perry, P.: Better Knowledge Management through Knowledge Engineering, Intelligent Systems, Volume 16, Number 1, 2005, pp. 36-43

[17] Richardson, M., Domingos, P.: Learning with Knowledge from Multiple Experts, in proc. of the Twentieth International Conference on Machine Learning, Washington, DC, Morgan Kaufmann, 2003, pp. 624-631 